\title{
Analysis of Combined Radiation and Forced Convection Heat Transfer in 3D Laminar Flow over an Inclined Forward Facing Step
}

\author{
A. Dehghani Rayeni, S. A. Gandjalikhan Nassab* \\ Department of Mechanical Engineering, Islamic Azad University, Kerman Branch, Kerman, Iran \\ Email: *Ganj110@uk.ac.ir
}

Received 15 June 2015; accepted 27 March 2016; published 30 March 2016

Copyright (C) 2016 by authors and Scientific Research Publishing Inc.

This work is licensed under the Creative Commons Attribution International License (CC BY). http://creativecommons.org/licenses/by/4.0/

c) (i) Open Access

\begin{abstract}
In the current study, a numerical investigation of three-dimensional combined convection-radiation heat transfer over an inclined forward facing step (FFS) in a horizontal rectangular duct is presented. The fluid is treated as a gray, absorbing, emitting and scattering medium. To simulate the incline surface of FFS, the blocked-off method is employed in this study. The set of governing equations for gas flow are solved numerically using the CFD technique to obtain the temperature and velocity fields. Since the gas is considered as a radiating medium, all of the convection, conduction and radiation heat transfer mechanisms are presented in the energy equation. For computation of radiative term in energy equation, the radiative transfer equation (RTE) is solved numerically by the discrete ordinates method (DOM) to find the divergence of radiative heat flux distribution inside the radiating medium. The effects of optical thickness, radiation-conduction parameter and albedo coefficient on heat transfer behavior of the system are carried out.
\end{abstract}

\section{Keywords}

3-D Laminar Convection Flow, Forward Facing Step, Radiation, DOM

\section{Introduction}

Forced convection flow over a backward or forward facing step in a three-dimensional channel is widely encountered in engineering applications. These geometries are important in industrial studies. In these types of convection flow, separating and reattaching regions exist because of the sudden changes in flow geometry. Separation flows accompanied with heat transfer are frequently encountered in many systems, such as cooling of

${ }^{*}$ Corresponding author.

How to cite this paper: Rayeni, A.D. and Nassab, S.A.G. (2016) Analysis of Combined Radiation and Forced Convection Heat Transfer in 3D Laminar Flow over an Inclined Forward Facing Step. Journal of Electronics Cooling and Thermal Control, 6, 1-18. http://dx.doi.org/10.4236/jectc.2016.61001 
electronic systems, power generating equipments, gas turbine blades, heat exchangers, combustion chamber and ducts flows used in industrial applications. In some of the mentioned advices, specially, when soot particles exist in the combustion product, the radiation effect may be important. Besides, the trend toward increasing temperature in modern technological systems has promoted concerted effort to develop more comprehensive and accurate theoretical methods to treat radiation. Therefore, for having more accurate and reliable results in the analysis of these types of flow, the flowing gas must be considered as a radiating medium and all of the heat transfer mechanisms including convection, conduction and radiation, must be taken into account. The flow over backward facing step (BFS) or forward facing step (FFS) has the most features of separated flows. Although the geometry of BFS or FFS flow is very simple, the heat transfer and fluid flow over these types of step contain most of complexities. Consequently, it has been used in the benchmark investigations. There are many studies about laminar convection flow over BFS in a 2-D duct by several investigators [1]-[4]. Velocity measurements were reported for three-dimensional laminar separated airflow adjacent to a backward-facing step using twocomponent laser Doppler velocimeter by Armaly et al. [5]. The results showed some interesting flow behaviors that could not be deduced from two-dimensional investigations. Beaudoin et al. [6] studied three-dimensional stationary structure of the flow over a backward-facing step experimentally. That study revealed that actually three-region of the two-dimensional flow was potentially unstable through the centrifugal instability. Iwai et al. [7] studied three-dimensional numerical simulation for flows over a backward-facing step at low Reynolds number in order to investigate the effects of the duct aspect ratio. In that study, distribution patterns of both Nusselt number and the skin friction coefficient on the bottom wall were paid. They reported that an aspect ratio of as large as $\mathrm{AR}=16$ at least was needed to obtain a 2-D region at the mid-plane for $\mathrm{Re}=250$. In a numerical research work by Nie and Armaly [8], distributions of wall temperature, Nusselt number and friction coefficient on all of the bounding walls of laminar three-dimensional forced convection flow adjacent to backward-facing step in a rectangular duct were reported. Beside of the above works, laminar convection flow over BFS in a 3-D duct was studied by several investigators [9]-[11]. Although there are many research studies about BFS geometries, the fluid flow with heat transfer over forward facing step (FFS) received less attention in comparison to the convection flow over BFS. In a recent study, Bahrami and Gandjalikhan Nassab [12] analyzed the convection flow over forward facing step in a duct to investigate the amount of entropy generation in this type of flow. A review of research on laminar convection flow over backward and forward facing step was done by Abu-Mulaweh [13]. In that study, a comprehensive review of such flows which have been reported in several studies in the open literature was presented. The purpose was to give a detailed summary of the effect of several parameters such as step height, Reynolds number, Prandtl number and the buoyancy force on the flow and temperature distributions downstream of the step. Also at different points of view, this geometry (FFS) was studied by several researchers [14] [15].

There are many engineering applications, in which the forward or backward-facing step is inclined. Simulations of three-dimensional laminar forced convection adjacent to inclined backward-facing step in rectangular duct were presented by Chen et al. [16] to examine the effects of step inclination on flow and heat transfer distributions. Velocity, temperature, Nusselt number and friction coefficient distributions were presented in that study. The effects of step inclination angle on Nusselt number and friction coefficient distributions were showed by plotting many figures. In a recent study, Gandjalikhan Nassab et al. [17] studied the turbulent forced convection flow adjacent to inclined forward facing step in a duct. In that study, the Navier-Stokes and energy equations were solved in the computational domain by computational fluid dynamic (CFD) method using conformal mapping technique. By this method, the effect of step inclination angle on flow and temperature distributions was determined. In all of the mentioned studies, the effect of radiative heat transfer in fluid flow was not studied, such that the gas energy equation only contains the convection and conduction terms. In a forced convection problem, when the flowing gas behaves as a participating medium, its complex absorption, emission and scattering introduce a considerable difficulty in the simulation of these flows. There are limited numbers of literatures about the radiative transfer problems in convection flows with complex 2-D and 3-D geometries.

Bouali and Mezrhab [18] studied heat transfer by laminar forced convection with considering surface radiation in a divided vertical channel with isotherm side walls. They found that the surface radiation has important effect on the Nusselt number in convective flow with high Reynolds numbers. Azad and Modest [19] investigated the problem of combined radiation and turbulent forced convection in absorbing, emitting and linearly anisotropic scattering gas particulate flow through a circular tube.

Simulations of laminar forced convection flow of a radiating gas adjacent to backward and forward facing 
steps in two-dimensional ducts under different conditions were presented in several studies [20]-[23]. The results of these works show that the radiative parameters have great effects on the thermal behaviors of the convective flow. In addition, 3-D numerical analysis of combined convection-radiation heat transfer over a BFS and a recess in horizontal ducts were studied by several researchers [24]-[26].

The study of mixed convection heat transfer in 3-D horizontal and inclined ducts with considering radiation effects has been numerically examined in detail by Chiu et al. [27] [28]. Those works were primarily focused on the interaction of the thermal radiation with mixed convection for a gray fluid in rectangular ducts. The vorticity-velocity method was employed to solve the three-dimensional Navier-Stokes equations while the integrodifferential radiative transfer equation was solved by the discrete ordinates method. Results revealed that radiation effects have a considerable impact on the heat transfer and would reduce the thermal buoyancy effects. Besides, it was revealed that the development of temperature was accelerated by the radiation effects.

Although there are limited studies about laminar forced convection flow of radiating gas over a BFS and FFS, but base on the author's knowledge, 3-D combined convection and radiation heat transfer over an inclined forward-facing step in a duct, is still not studied by DOM and block-off method. Therefore, the present research work deals the three-dimensional simulations of incompressible laminar forced convection flow of a radiating gas over an inclined forward facing step in rectangular duct, while the well known DOM and block-off method are employed to solve the radiation problem.

\section{Problem Description}

Three-dimensional combined convection and radiation heat transfer in a horizontal heated rectangular duct with an inclined forward facing step is numerically simulated. Schematic of the computational domain is shown in Figure 1. Fluid enters the duct with uniform velocity $U_{\text {。 }}$ and uniform temperature $T_{i n}$. All of the duct's walls are kept at a constant temperature $T_{w}$ which is greater than the fluid inlet temperature. The upstream and downstream heights of the duct are $H$ and $h$, respectively, such that this geometry provides the step height of $s$, with contraction ratio $(C R=h / H)$ of 0.5 . The width of the duct is $D$ with an aspect ratio of $A R=D / h$, which is considered equal to 4 in the present computations. The upstream length of the duct is considered to be $L_{1}=5 H$ and the rest of the channel length is equal to $L_{2}=15 \mathrm{H}$. This is made to ensure that the flow at the inlet and outlet sections is not affected significantly by the sudden changes in the geometry and flow at exit section becomes fully developed. Also, the step inclination angle indicated by $\Phi$ is considered to be $45^{\circ}$ in all subsequent calculations.

\section{Basic Equation}

For incompressible, steady and three-dimensional laminar flow, the governing equations are the conservations of mass, momentum and energy that can be written as follows:

$$
\begin{gathered}
\frac{\partial u}{\partial x}+\frac{\partial v}{\partial y}+\frac{\partial w}{\partial z}=0 \\
u \frac{\partial u}{\partial x}+v \frac{\partial u}{\partial y}+w \frac{\partial u}{\partial z}=-\frac{1}{\rho} \frac{\partial p}{\partial x}+\frac{\mu}{\rho}\left(\frac{\partial^{2} u}{\partial x^{2}}+\frac{\partial^{2} u}{\partial y^{2}}+\frac{\partial^{2} u}{\partial z^{2}}\right)
\end{gathered}
$$

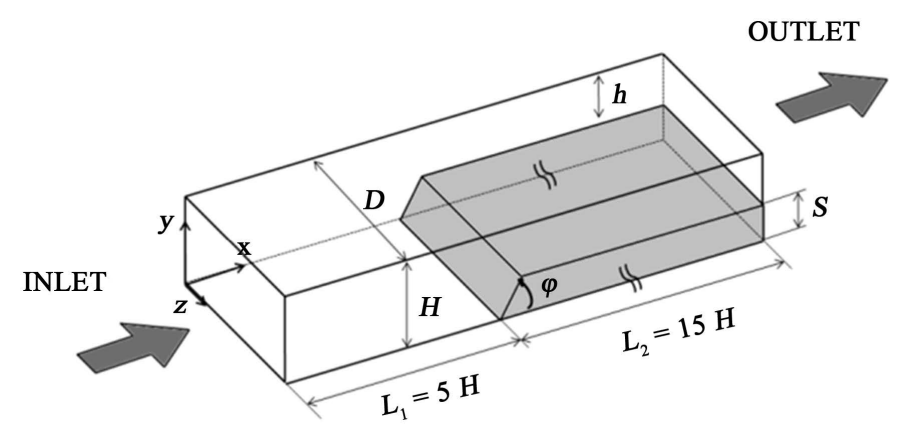

Figure 1. Schematic of computational domain. 


$$
\begin{gathered}
u \frac{\partial v}{\partial x}+v \frac{\partial v}{\partial y}+w \frac{\partial v}{\partial z}=-\frac{1}{\rho} \frac{\partial p}{\partial y}+\frac{\mu}{\rho}\left(\frac{\partial^{2} v}{\partial x^{2}}+\frac{\partial^{2} v}{\partial y^{2}}+\frac{\partial^{2} v}{\partial z^{2}}\right) \\
u \frac{\partial w}{\partial x}+v \frac{\partial w}{\partial y}+w \frac{\partial w}{\partial z}=-\frac{1}{\rho} \frac{\partial p}{\partial z}+\frac{\mu}{\rho}\left(\frac{\partial^{2} w}{\partial x^{2}}+\frac{\partial^{2} w}{\partial y^{2}}+\frac{\partial^{2} w}{\partial z^{2}}\right) \\
\frac{\partial}{\partial x}\left(\rho u c_{p} T\right)+\frac{\partial}{\partial y}\left(\rho v c_{p} T\right)+\frac{\partial}{\partial z}\left(\rho w c_{p} T\right) \\
=\kappa\left(\frac{\partial^{2} T}{\partial x^{2}}+\frac{\partial^{2} T}{\partial y^{2}}+\frac{\partial^{2} T}{\partial z^{2}}\right)-\nabla \cdot \boldsymbol{q}_{r}
\end{gathered}
$$

In the above equations, $u, v$ and $w$ are the velocity components in $x$-, $y$ - and $z$-directions, respectively; $\rho$ the density; $p$ the pressure; $T$ the temperature; $\mu$ the dynamic viscosity; $c_{p}$ the specific heat; $\kappa$ the thermal conductivity and $\boldsymbol{q}_{r}$ is the radiative flux vector.

The boundary conditions are treated as no slip condition at the solid walls (zero velocity) and constant temperature of at all boundary surfaces. At the inlet duct section, the flow has uniform velocity of with uniform temperature of $T_{i n}$, which is assumed to be lower than. At the outlet section, zero axial gradients for velocity components and gas temperature are employed.

\subsection{Gas Radiation Modeling}

In the energy equation, besides the convective and conductive terms, the radiative term as the divergence of the radiative heat flux, i.e. $\nabla \cdot \boldsymbol{q}_{r}$ also presented. This radiative term can be computed as follow [29]:

$$
\nabla \cdot \boldsymbol{q}_{r}=\sigma_{a}\left(4 \pi I_{b}(\boldsymbol{r})-\int_{4 \pi} I(\boldsymbol{r}, \boldsymbol{s}) \mathrm{d} \Omega\right)
$$

In the above equation, $I(\boldsymbol{r}, \boldsymbol{s})$ is the radiation intensity at the situation $\boldsymbol{r}$ and in the direction $\boldsymbol{s}$ and $I_{b}(\boldsymbol{r})=\frac{\sigma(T(\boldsymbol{r}))^{4}}{\pi}$ is the black body radiation intensity where $\sigma_{a}$ is the absorption coefficient. For calculation of $\nabla \cdot \boldsymbol{q}_{r}$, the radiation intensity field is primary needed. To obtain this term, it is necessary to solve the radiative transfer equation. This equation for an absorbing, emitting and scattering gray medium can be expressed as [29]:

$$
(\boldsymbol{s} \cdot \nabla) I(\boldsymbol{r}, \boldsymbol{s})=-\beta I(\boldsymbol{r}, \boldsymbol{s})+\sigma_{a} I_{b}(\boldsymbol{r})+\frac{\sigma_{s}}{4 \pi} \int_{4 \pi} I(\boldsymbol{r}, \boldsymbol{s}) \phi\left(\boldsymbol{s}, \boldsymbol{s}^{\prime}\right) \mathrm{d} \Omega^{\prime}
$$

where $\sigma_{s}$ is the scattering coefficient; $\beta=\sigma_{a}+\sigma_{s}$ is the extinction coefficient and $\phi\left(s, s^{\prime}\right)$ is the scattering phase function for the radiation from incoming direction $s^{\prime}$ and confined within the solid angle $\mathrm{d} \Omega^{\prime}$ to scattered direction $s$ confined within the solid angle $\mathrm{d} \Omega$. In this study, the phase function is equal to unity because the assumption of isotropic scattering medium. The boundary condition for a diffusely emitting and reflecting gray wall is:

$$
I\left(\boldsymbol{r}_{w}, \boldsymbol{s}\right)=\varepsilon_{w} I_{b}\left(\boldsymbol{r}_{w}\right)+\frac{\left(1-\varepsilon_{w}\right)}{\pi} \int_{\boldsymbol{n}_{w} \cdot s^{\prime}<0} I\left(\boldsymbol{r}_{w}, \boldsymbol{s}\right)\left|\boldsymbol{n}_{w} \cdot \boldsymbol{s}^{\prime}\right| \mathrm{d} \Omega^{\prime} \quad \boldsymbol{n}_{w} \cdot \boldsymbol{s}>0
$$

in which $\varepsilon_{w}$ is the wall emissivity; $I_{b}\left(\boldsymbol{r}_{w}\right)$ is the black body radiation intensity at the temperature of the boundary surface and $\boldsymbol{n}_{w}$ is the outward unit vector normal to the surface. Since, the RTE depends on the temperature fields through the emission term $I_{b}\left(\boldsymbol{r}_{w}\right)$, thus it must be solved simultaneously with overall energy equation. RTE is an integro-differential equation that can be solved with discrete ordinates method.

In the DOM, Equation (7) is solved for a set of $\mathrm{n}$ different directions, $\boldsymbol{s}_{i}, i=1,2,3, \cdots, n$ and integrals over solid angle are replaced by the numerical quadrature, that is,

$$
\int_{4 \pi} f(s) \mathrm{d} \Omega \cong \sum_{i=1}^{n} w_{i} f\left(s_{i}\right)
$$


where $w_{i}$ are the quadrature weights associated with the directions $\boldsymbol{s}_{i}$. Thus, according to this method, Equation (7) is approximated by a set of $\mathrm{n}$ equations, as follows:

$$
\left(\boldsymbol{s}_{i} \cdot \nabla\right) I\left(\boldsymbol{r}, \boldsymbol{s}_{i}\right)=-\beta I\left(\boldsymbol{r}, \boldsymbol{s}_{i}\right)+\sigma_{a} I_{b}(\boldsymbol{r})+\frac{\sigma_{s}}{4 \pi} \sum_{j=1}^{n} I\left(\boldsymbol{r}, \boldsymbol{s}_{j}\right) \phi\left(\boldsymbol{s}_{j}, \boldsymbol{s}_{i}\right) w_{j} \quad i=1,2,3, \cdots, n
$$

subjected to the boundary conditions:

$$
I\left(\boldsymbol{r}_{w}, \boldsymbol{s}_{i}\right)=\varepsilon_{w} I_{b}\left(\boldsymbol{r}_{w}\right)+\frac{\left(1-\varepsilon_{w}\right)}{\pi} \sum_{\boldsymbol{n}_{w} \cdot s_{j}<0} I\left(\boldsymbol{r}_{w}, \boldsymbol{s}_{j}\right)\left|\boldsymbol{n}_{w} \cdot \boldsymbol{s}_{j}\right| w_{j} \quad \boldsymbol{n}_{w} \cdot \boldsymbol{s}_{i}>0
$$

Also $\nabla \cdot \boldsymbol{q}_{r}$ is represented as:

$$
\nabla \cdot \boldsymbol{q}_{r}=\sigma_{a}\left(4 \pi I_{b}(\boldsymbol{r})-\sum_{i=1}^{n} I\left(\boldsymbol{r}, \boldsymbol{s}_{i}\right) w_{i}\right)
$$

At any arbitrary surface, heat flux may also be determined from the surface energy balance as:

$$
\boldsymbol{q} \cdot n\left(r_{w}\right)=\varepsilon_{w}\left(\pi I_{b}\left(r_{w}\right)-\sum_{n_{w} \cdot s_{j}<0} I_{i}\left(r_{w}\right)\left|n_{w} \cdot s_{i}\right| w_{i}\right)
$$

In 3-D Cartesian coordinate system, Equation (10) becomes as follows [29]:

$$
\xi_{i} \frac{\partial I_{i}}{\partial x}+\eta_{i} \frac{\partial I_{i}}{\partial y}+\mu_{i} \frac{\partial I_{i}}{\partial z}+\beta I_{i}=\beta S_{i} \quad i=1,2,3, \cdots, n
$$

where

$$
S_{i}=(1-\omega) I_{b}+\frac{\omega}{4 \pi} \sum_{j=1}^{n} I\left(\boldsymbol{r}, \boldsymbol{s}_{j}\right) \phi\left(\boldsymbol{s}_{j}, \boldsymbol{s}_{i}\right) w_{j} \quad i=1,2,3, \cdots, n
$$

In fact $S_{i}$ is a shorthand for the radiative source function. In Equation (15), $\omega$ is the albedo coefficient, defined as $\omega=\frac{\sigma_{s}}{\beta}$. The finite difference form of Equation (14) gives the following form for radiant intensity [29]:

$$
I_{p i}=\frac{\left|\xi_{i}\right| A_{x} I_{x i} i / \gamma_{x}+\left|\eta_{i}\right| A_{y} I_{y i} i / \gamma_{y}+\left|\mu_{i}\right| A_{z} I_{z i} i / \gamma_{z}+\beta \forall S_{p i}}{\beta \forall+\left|\xi_{i}\right| A_{x} / \gamma_{x}+\left|\eta_{i}\right| A_{y} / \gamma_{y}+\left|\mu_{i}\right| A_{z} / \gamma_{z}}
$$

in which $\xi_{i}, \eta_{i}$ and $\mu_{i}$ are the direction cosines for the direction $s_{i}$ and $\forall$ is the element cell volume.

The details of the numerical solution of RTE by DOM were also described in the previous work by the second author, in which the thermal characteristics of porous radiant burners were investigated [30].

For the radiative boundary conditions, the walls are assumed to emit and reflect diffusely with constant wall emissivity, $\varepsilon_{w}=0.8$. In addition, the inlet and outlet sections are considered for radiative transfer as black walls at the fluid temperatures in inlet and outlet sections, respectively.

\subsection{Non-Dimensional Forms of the Governing Equations}

In numerical solution of the set of governing equations including the continuity, momentum and energy, the following dimensionless parameters are used to obtain the non dimensional forms of these equations:

$$
\begin{aligned}
& (X, Y, Z)=\left(\frac{x}{H}, \frac{y}{H}, \frac{z}{H}\right),(U, V, W)=\left(\frac{u}{U_{\circ}}, \frac{v}{U_{\circ}}, \frac{w}{U_{\circ}}\right), \\
& v_{w}^{*}=\frac{v_{w}}{U_{\circ}}, P=\frac{p}{\rho U_{\circ}^{2}}, \Theta=\frac{T-T_{i n}}{T_{w}-T_{i n}}, \theta_{1}=\frac{T_{i n}}{T_{w}-T_{i n}}, \theta_{2}=\frac{T_{w}}{T_{i n}}, \\
& I^{*}=\frac{I}{\sigma T_{w}^{4}}, S^{*}=\frac{S}{\sigma T_{w}^{4}}, \tau=\beta \cdot H,(1-\omega)=\frac{\sigma_{a}}{\beta}, \operatorname{Pr}=\frac{v}{\alpha}, \\
& \operatorname{Re}=\frac{\rho U_{0} H}{\mu}, P e=\operatorname{Re} \cdot \operatorname{Pr}, R C=\frac{\sigma T_{w}^{3} H}{k}, q_{r}^{*}=\frac{q_{r}}{\sigma T_{w}^{4}}
\end{aligned}
$$


The non-dimensional forms of the governing equations are as follows:

$$
\begin{gathered}
\frac{\partial U}{\partial X}+\frac{\partial V}{\partial Y}+\frac{\partial W}{\partial Z}=0 \\
\frac{\partial}{\partial X}\left(U^{2}-\frac{1}{\operatorname{Re}} \frac{\partial U}{\partial X}\right)+\frac{\partial}{\partial Y}\left(U V-\frac{1}{\operatorname{Re}} \frac{\partial U}{\partial Y}\right)+\frac{\partial}{\partial Z}\left(U W-\frac{1}{\operatorname{Re}} \frac{\partial U}{\partial Z}\right)=-\frac{\partial P}{\partial X} \\
\frac{\partial}{\partial X}\left(U V-\frac{1}{\operatorname{Re}} \frac{\partial V}{\partial X}\right)+\frac{\partial}{\partial Y}\left(V^{2}-\frac{1}{\operatorname{Re}} \frac{\partial V}{\partial Y}\right)+\frac{\partial}{\partial Z}\left(V W-\frac{1}{\operatorname{Re}} \frac{\partial V}{\partial Z}\right)=-\frac{\partial P}{\partial Y} \\
\frac{\partial}{\partial X}\left(U W-\frac{1}{\operatorname{Re}} \frac{\partial W}{\partial X}\right)+\frac{\partial}{\partial Y}\left(V W-\frac{1}{\operatorname{Re}} \frac{\partial W}{\partial Y}\right)+\frac{\partial}{\partial Z}\left(W^{2}-\frac{1}{\operatorname{Re}} \frac{\partial W}{\partial Z}\right)=-\frac{\partial P}{\partial Z} \\
\frac{\partial}{\partial X}\left(U \Theta-\frac{1}{P e} \frac{\partial \Theta}{\partial X}\right)+\frac{\partial}{\partial Y}\left(V \Theta-\frac{1}{P e} \frac{\partial \Theta}{\partial Y}\right)+\frac{\partial}{\partial Z}\left(W \Theta-\frac{1}{P e} \frac{\partial \Theta}{\partial Z}\right) \\
+\frac{\tau(1-\omega) R C \theta_{1} \theta_{2}}{P e}\left[\frac{4}{\theta_{2}^{4}}\left(\frac{\Theta}{\theta_{1}}+1\right)^{4}-\sum_{i=1}^{n} I_{i}^{*} w_{i}\right]=0
\end{gathered}
$$

\subsection{The Main Physical Quantities}

The main physical quantities of interest in heat transfer study are the mean bulk temperature and Nusselt number. The mean bulk temperature along the channel was calculated using the following equation:

$$
\Theta_{b}=\frac{\int_{0}^{2} \int_{0}^{1} \Theta U \mathrm{~d} Y \mathrm{~d} Z}{\int_{0}^{2} \int_{0}^{1} U \mathrm{~d} Y \mathrm{~d} Z}
$$

In the combined convection-radiation heat transfer, the energy transport from the duct wall to the gas flow depends on two related factors:

1. Fluid temperature gradient on the wall;

2. Rate of radiative heat exchange on boundary surface.

Therefore, total heat flux on the wall is the sum of convective and radiative heat fluxes such that $q_{t}=q_{c}+q_{r}=-k\left(\frac{\partial T}{\partial y}\right)+q_{r}$.

Therefore, the function of total Nusselt number $\left(N u_{t}=\frac{q_{t} H}{k\left(T_{W}-T_{M}\right)}\right)$ is the sum of local convective Nusselt number $\left(N u_{c}\right)$, and local radiative Nusselt number $\left(N u_{r}\right)$.

Total Nusselt numbers is given as follows [22]:

$$
N u_{t}=N u_{c}+N u_{r}=\left.\frac{-1}{\Theta_{w}-\Theta_{M}} \frac{\partial \Theta}{\partial Y}\right|_{Y=0}+\frac{R C \cdot \theta_{1} \cdot \theta_{2}}{\Theta_{w}-\Theta_{M}} q_{r}^{*}
$$

where

$$
\Theta_{M}=\frac{\int_{0}^{1} \Theta U \mathrm{~d} Y}{\int_{0}^{1} U \mathrm{~d} Y}
$$

Equation (24) contains two parts. The first term on the right hand side represents the convective Nusselt number, whereas the second term is the radiative Nusselt number. It should be considered that for pure convective hat transfer, total Nusselt number is equal to the convective one. 


\section{Numerical Procedure}

Finite difference forms of the continuity, momentum and energy equations ((18) to (22)) were obtained by integrating over an elemental cell volume with staggered control volumes for the $\mathrm{x}-, \mathrm{y}$ - and $\mathrm{z}$ - velocity components. Other variables of interest were computed at the grid nodes.

The discretized forms of the governing equations were numerically solved by the SIMPLE algorithm of Patankar and Spalding [31]. Numerical solutions were obtained iteratively by the line-by-line method. Numerical calculations were performed by writing a computer program in FORTRAN. Based on the result of grid tests for obtaining the grid-independent solutions, six different meshes were used in the grid independence study. Numerical solutions are obtained iteratively such that iterations are terminated when sum of the absolute residuals is less than $10^{-4}$ for momentum and energy equations. But in the numerical solution of RTE, the maximum difference between the radiative intensities computed during two consecutive iteration levels did not exceed $10^{-6}$ at each nodal point for the converged solution. By this numerical strategy, the velocity, temperature and radiation intensity distributions inside the flow domain can be obtained. The corresponding maximum values of convective and radiative Nusselt numbers along the centerline on the bottom wall are calculated and tabulated in Table 1 . As it is seen, a grid size of $360 \times 36 \times 36$ can be chosen for obtaining the grid independent solution, such that the subsequent numerical calculations are made based on this grid size. It should be mentioned that near the top, bottom and step walls clustering is employed in the $\mathrm{x}$ - and $\mathrm{y}$ - directions for obtaining more accuracy in the numerical calculations. To simulate the incline surface in the computational domain, the blocked off method is used in this study (see Figure 2). Also, for computation the divergence of radiative heat flux, which is needed for the numerical solution of the energy equation by DOM, $\mathrm{S}_{4}$ approximation has been used in this study.

\begin{tabular}{|l|l|l|l|l|l|l|l|l|l|l|l|l|l|l|l|l|l|l|l|l|l|}
\hline 1 & 1 & 1 & 1 & 1 & 1 & 1 & 1 & 1 & 1 & 1 & 1 & 1 & 1 & 1 & 1 & 1 & 1 & 1 & 1 & 1 & 1 \\
\hline 1 & 1 & 1 & 1 & 1 & 1 & 1 & 1 & 1 & 1 & 1 & 1 & 1 & 1 & 1 & 1 & 1 & 1 & 1 & 1 & 1 & 1 \\
\hline 1 & 1 & 1 & 1 & 1 & 1 & 1 & 1 & 1 & 1 & 1 & 1 & 1 & 1 & 1 & 1 & 1 & 1 & 1 & 1 & 1 & 1 \\
\hline 1 & 1 & 1 & 1 & 1 & 1 & 1 & 1 & 1 & 1 & 1 & 1 & 1 & 1 & 1 & 1 & 1 & 1 & 1 & 1 & 1 & 1 \\
\hline 1 & 1 & 1 & 1 & 1 & 1 & 1 & 1 & 1 & 1 & 1 & 1 & 1 & 1 & 1 & 1 & 1 & 1 & 1 & 1 & 1 & 1 \\
\hline 1 & 1 & 1 & 1 & 1 & 1 & 1 & 1 & 1 & 1 & 1 & 1 & 1 & 1 & 1 & 1 & 1 & 1 & 1 & 1 & 1 & 1 \\
\hline 1 & 1 & 1 & 1 & 1 & 1 & 1 & 1 & 1 & 1 & 1 & 1 & 0 & 0 & 0 & 0 & 0 & 0 & 0 & 0 & 0 & 0 \\
\hline 1 & 1 & 1 & 1 & 1 & 1 & 1 & 1 & 1 & 1 & 1 & 0 & 0 & 0 & 0 & 0 & 0 & 0 & 0 & 0 & 0 & 0 \\
\hline 1 & 1 & 1 & 1 & 1 & 1 & 1 & 1 & 1 & 1 & 0 & 0 & 0 & 0 & 0 & 0 & 0 & 0 & 0 & 0 & 0 & 0 \\
\hline 1 & 1 & 1 & 1 & 1 & 1 & 1 & 1 & 1 & 7 & 0 & 0 & 0 & 0 & 0 & 0 & 0 & 0 & 0 & 0 & 0 & 0 \\
\hline 1 & 1 & 1 & 1 & 1 & 1 & 1 & 1 & 1 & 0 & 0 & 0 & 0 & 0 & 0 & 0 & 0 & 0 & 0 & 0 & 0 & 0 \\
\hline 1 & 1 & 1 & 1 & 1 & 1 & 1 & 1 & 0 & 0 & 0 & 0 & 0 & 0 & 0 & 0 & 0 & 0 & 0 & 0 & 0 & 0 \\
\hline 1 & 1 & 1 & 1 & 1 & 1 & 1 & 0 & 0 & 0 & 0 & 0 & 0 & 0 & 0 & 0 & 0 & 0 & 0 & 0 & 0 & 0 \\
\hline
\end{tabular}

Figure 2. Blocked-off region in a regular grid.

Table 1. Grid independence study, $R=250, R C=100, \omega=0.5, \tau=0.005$.

\begin{tabular}{ccc}
\hline Grid size & Value of the maximum convective Nusselt number & Value of the maximum radiative Nusselt number \\
\hline $100 \times 15 \times 15$ & 22.351 & - \\
$160 \times 20 \times 20$ & 26.354 & 16.738 \\
$240 \times 25 \times 25$ & 29.172 & 18.957 \\
$300 \times 30 \times 30$ & 31.259 & 20.554 \\
$360 \times 36 \times 36$ & 32.183 & 21.275 \\
$420 \times 40 \times 40$ & 32.301 & 21.352 \\
\hline
\end{tabular}




\section{Blocked-Off Method}

In many cases, a computer program written for a regular grid can be improved to handle an irregularly shaped computational domain using the blocked-off method [32]. In this technique, the whole 3-D region is divided into two parts: active and inactive or blocked-off regions. The region where solutions are done is known as the active region and the remaining portion is known as the inactive or the blocked-off region. Therefore, by rendering inactive some of the control volumes of the regular grid, the remaining active control volumes form the desired irregular domain with complex boundary. By this technique, the surface of inclined step in the present analysis is approximated by a series of fine rectangular steps, Figure 2. It should be mentioned that the control volumes, which are inside the active region, are designated as 1 and otherwise they are 0, as shown in Figure 2. It is obvious that using fine grids in the interface region between active and inactive zones causes to have an approximated boundary which is more similar to the true boundary.

According to the blocked-off technique, known values of the dependent variables must be established in all inactive control volumes. If the inactive region represents a stationary solid boundary as in the case, the velocity components in that region must be equal to zero, and if the region is regarded as isothermal boundary, the known temperature must be established in the inactive control volumes.

\section{Code Validation}

As it was mentioned before, there are limited theoretical and experimental research works about heat transfer in laminar forced convection flow over FFS. First, the present numerical implementation was validated by reproducing the results of Iwai et al. [7] in which a forced convection gas flow over a BFS in a three-dimensional duct was studied. An attempt is made to compare the variation of Nusselt number along the centerline on the bottom wall with that obtained by Iwai et al. [7] in the cases of $E R=2$ and $A R=4$. The results are presented graphically in Figure 3. In this test case, at the upstream boundary, inlet flow is assumed to be fully developed. Other velocity components $\mathrm{V}$ and $\mathrm{W}$ are set to zero at the inlet, while the fluid is assumed to have a uniform temperature at this section. The side walls are treated adiabatic, while the other walls are isotherm with uniform temperature $T_{w}$ which is greater than the fluid inlet temperature. It is seen from Figure 3 that the value of Nusselt number increases downstream the step corner after which $N u_{\max }$ occurs near to the reattachment point, and then approaches to a constant value far from the step location. However, Figure 3 shows a good consistency between the present numerical results with those reported by Iwai et al. [7]. It should be mentioned that as the radiating effect of the gas flow is neglected in the study by Iwai et al. [7], therefore, the optical thickness of the media is set equal to zero in the computations of Figure 3.

To validate the present calculations in solving the RTE by DOM using the block-off method, there is not any theoretical result in combined convection and radiation heat transfer over an inclined forward facing step (FFS)

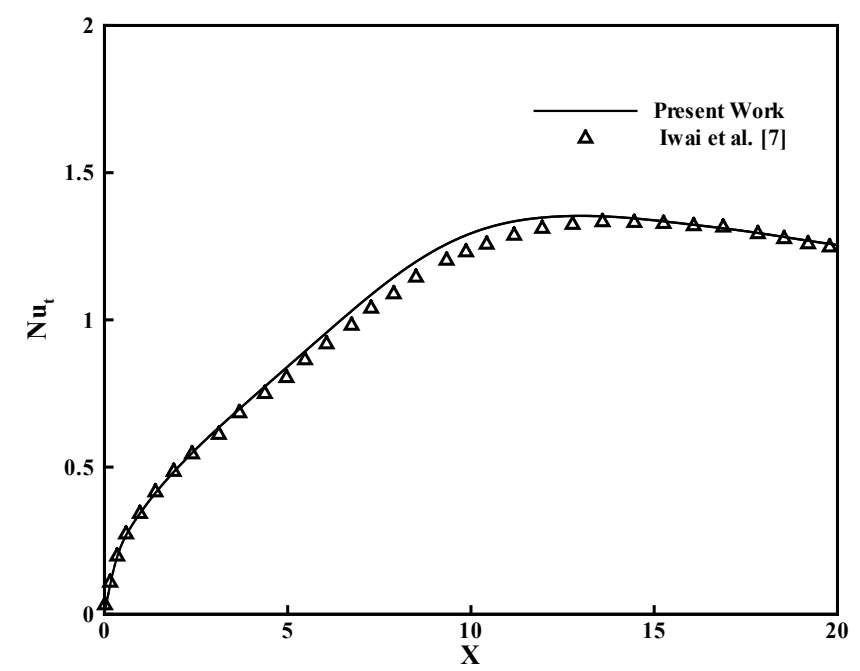

Figure 3. Comparison of numerical result for distribution of total Nusselt number along the centerline on the bottom wall in pure convection flow. 
in a three-dimensional duct. But there is a research work about two-dimensional laminar forced convection of radiating gas flow over an inclined FFS by Ansari and Gandjalikhan Nassab [22].

Therefore, second validation is done based on the results of Ref. [21] in which a 2-D laminar forced convection of gas flow was analyzed. In that work, the block-off method was considered for simulate the incline surface, with inclination angle of $\Phi=45$. Distributions of mean bulk temperature and convective Nusselt number are compared with that presented by Ansari and Gandjalikhan Nassab [22]. The results are presented graphically in Figure 4. As it is seen from this figure, a good agreement exists between the present numerical results with those obtained theoretically in Ref. [22].

\section{Results and Discussion}

In this study, numerical results are presented for combined convection and radiation heat transfer over an inclined FFS in a three -dimensional duct with a contraction ratio of $C R=0.5$ and aspect ratio of $A R=4$ at different conditions. The fixed inclination angle of $\Phi=45^{\circ}$ is considered for FFS. In numerical calculations, the Reynolds number is equal to 250 , while the Prandtl number is kept constant at 0.71 to guarantee the constant fluid physical properties.



(a)

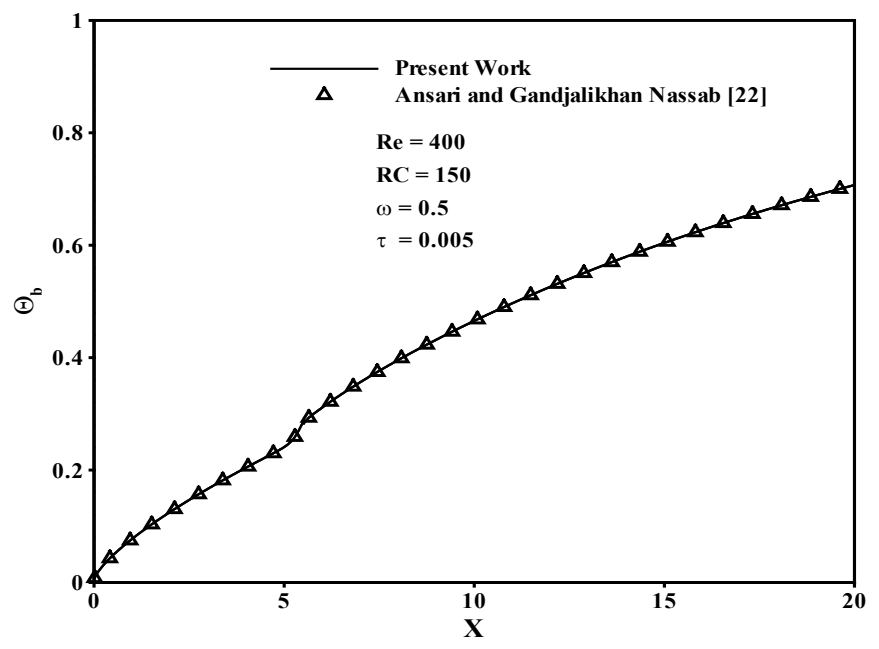

(b)

Figure 4. Comparison of numerical results with theoretical findings by other investigators. (a) Distribution of convective Nusselt number along the duct; (b) Distribution of convective Nusselt number along the duct. 


\subsection{The Effect of Radiation Heat Transfer in Forced Convection Flow of Radiating Gas}

In order to illustrate the radiation effect in thermal behavior of a convection flow, contours of total Nusselt number on the bottom wall with and without considering the radiation term in energy equation are presented in Figure 5. Figure 5(a) which is due to convection flow of a transparent fluid presents that the total Nusselt number starts from a high value at the inlet section $(X=0)$, which is due to the high heat flux encountered in the development of thermal boundary layer. Then, the Nusselt number decreases along the flow direction such that the value of Nusselt number nearly becomes zero closed to the step corner on the bottom wall where the fluid is at rest. After this point, there is a sharp increase in the convection coefficient as the flow passes over the inclined step which is due to the impact of relatively cooler fluid on the heated step surface and also due to the flow mixing in that region. After the inclined step, the value of local Nusselt number decreases and approaches to a constant value as the distance continuous to increase in the stream wise direction.

The effect of radiation in $\mathrm{Nu}$ distribution can be found if one compares Figure 5(a) and Figure 5(b) with each other. Figure 5(a) and Figure 5(b) illustrate similar pattern for distribution of convection coefficient, but, it can be seen that the radiative effect increases the value of total Nusselt number especially near to the duct walls, because of the surface radiation. Also, it should be mentioned that in combined convection-radiation heat transfer, total Nusselt number has a peak value before the FFS. This behavior will be explained in the explanation of the next figure. In order to show more clearly the variation of convection coefficient on the heated wall, the distribution of total Nusselt numbers on the bottom wall in the mid-plane of the duct $\left(z=\frac{D}{2}\right)$ is plotted in Figure 6, for two different cases (with and without considering the radiation effect). This figure shows the same thermal behavior for the convective flow as it was presented before in Figure 5. As it is seen from Figure 6 and
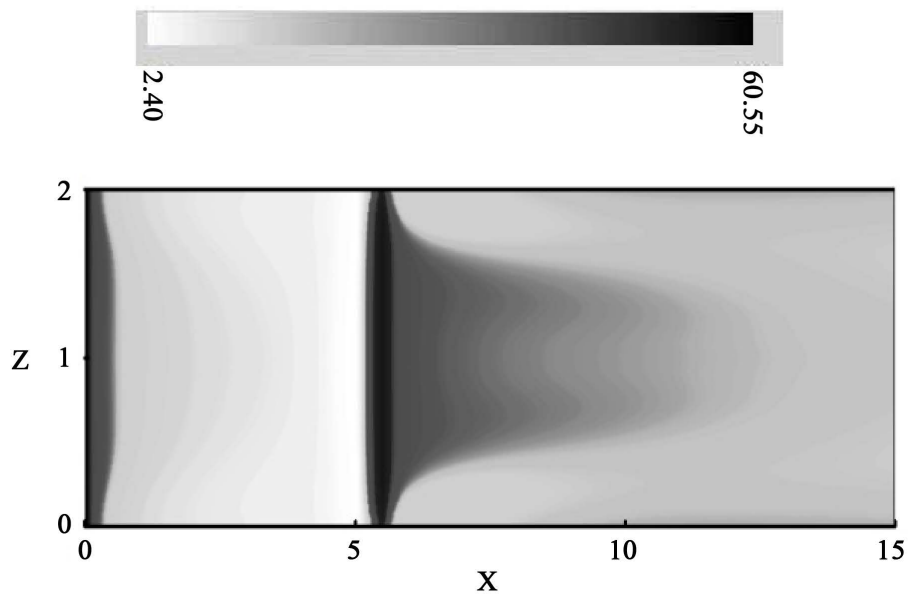

(a)

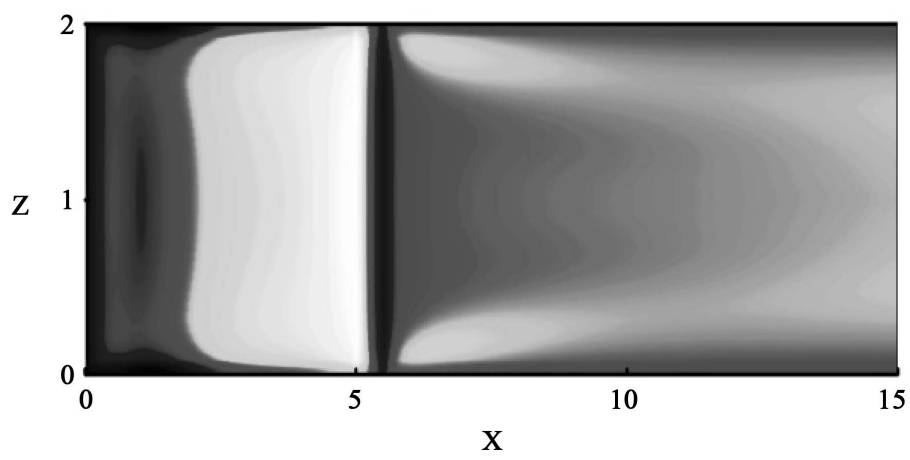

(b)

Figure 5. Distribution of total Nusselt number contours on the bottom wall, $R C=50, \omega=$ $0.5, \tau=0.005$. (a) without radiation effect; (b) with radiation effect. 


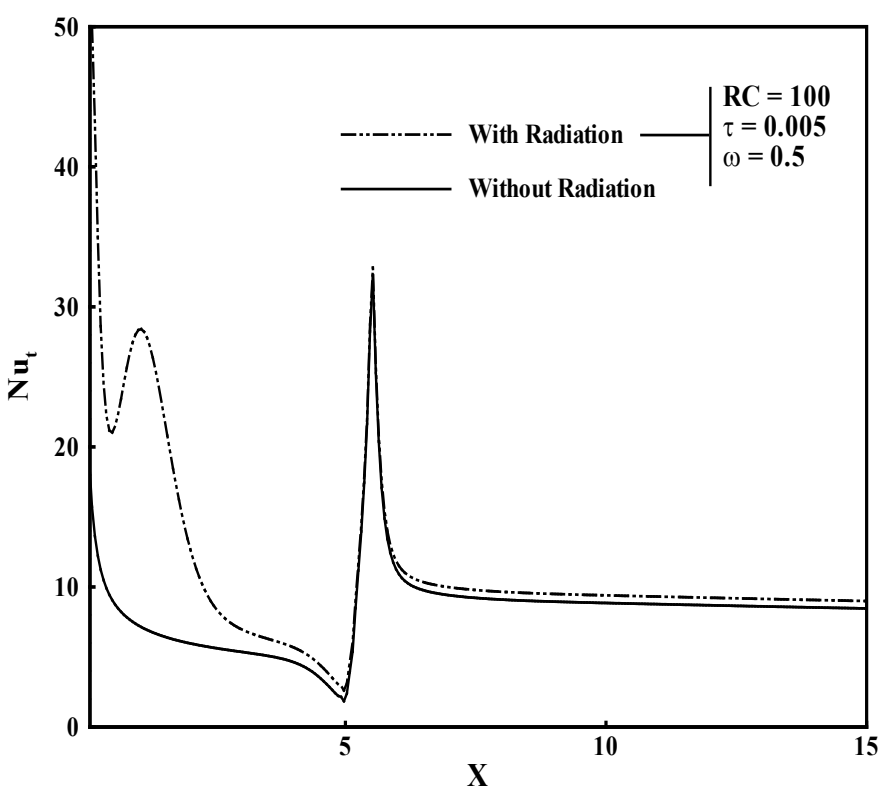

Figure 6. Distribution of total Nusselt numbers on the bottom wall in the duct mid-plane.

also from Figure 5, in the case of radiating gas flow, a local maximum Nusselt number takes place upstream to the step location. This behavior is due to the incoming radiant heat flux from the step surface towards the bottom wall that affects directly the radiative Nusselt number. It is obvious that as we move in downstream direction along the bottom wall towards the step, the rate of incoming radiation from the step surface on the bottom wall increases that causes a decreases in the radiative Nusselt number and then in $N u_{t}$. On the step surface, the amount of total Nusselt number suddenly increases after which $N u_{t}$ decreases and then approaches to a constant value far from the step.

In the convection flow of a radiating fluid, the optical thickness $(\tau)$, the radiation-conduction parameter $(R C)$ and the albedo coefficient $(\omega)$ are the main parameters that affect the thermal behavior of the radiation-convection system. In the next sections, an attempt is made to study the effects of these parameters on thermal behavior of the thermal system.

\subsection{The Effect of Optical Thickness}

A well-known radiation property and one of the important parameter in participating medium is the optical thickness that affects the temperature distribution inside the participating medium. High optical thickness means that the medium has great ability to absorb and emit radiant energy. For the convective flow with radiating heat transfer in the channel including an inclined 3-D forward facing step, as shown in Figure 1, the contours of convective, radiative and total Nusselt numbers along the bottom wall at different values of the optical thickness are presented in Figures 7-9, respectively.

Figure 7(a) and Figure 7(b) show that convective Nusselt number has a very small decrease by increasing in optical thickness. Because by increasing in the optical thickness, the amount of temperature difference inside the flow domain decreases that leads to decrease in temperature gradient on the heated walls. However, it can be concluded that the optical thickness has a small effect in convective Nusselt number, such that increasing in $\tau$ leads to small decrease in $N u_{c}$, as it seen from Figure 7. But a different trend is seen from Figure 8 for the variation of radiative Nusselt number with optical thickness. This figure shows that $N u_{r}$ starts from a high value at the inlet section, then $N u_{r}$ decrease and after having a peak value upstream the step, $N u_{r}$ decreases until it reaches to a constant value as the distance continues to increase in the stream-wise direction. Furthermore, Figure 8(a) and Figure 8(b) show that the radiative Nusselt number increases by increasing in optical thickness, which is due to the increase in bottom wall's outgoing radiative heat flux.

It is seen that optical thickness has a greater influence on the radiative Nusselt number than the convective 

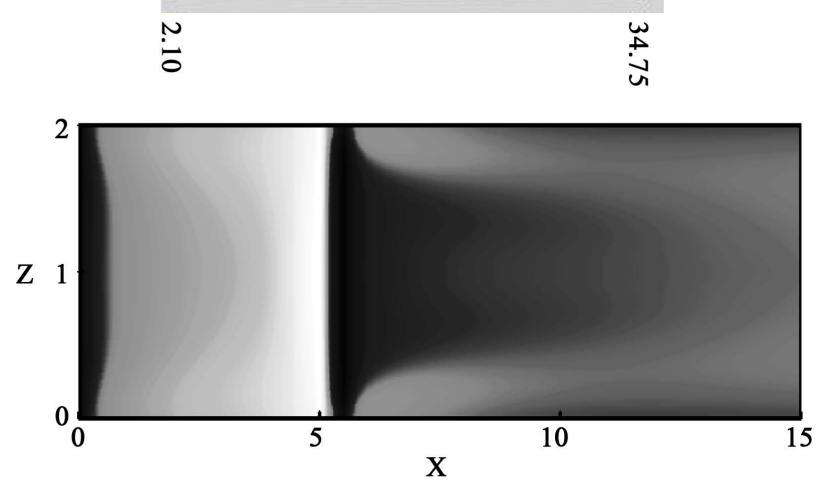

(a)

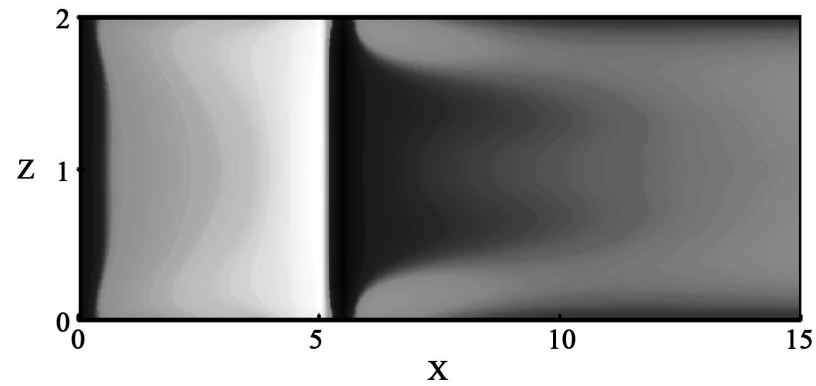

(b)

Figure 7. Distribution of the convective Nusselt number contours on the bottom wall at different optical thickness, $R C=100, \omega=0.5$. (a) $\tau=0.01$; (b) $\tau=0.05$.
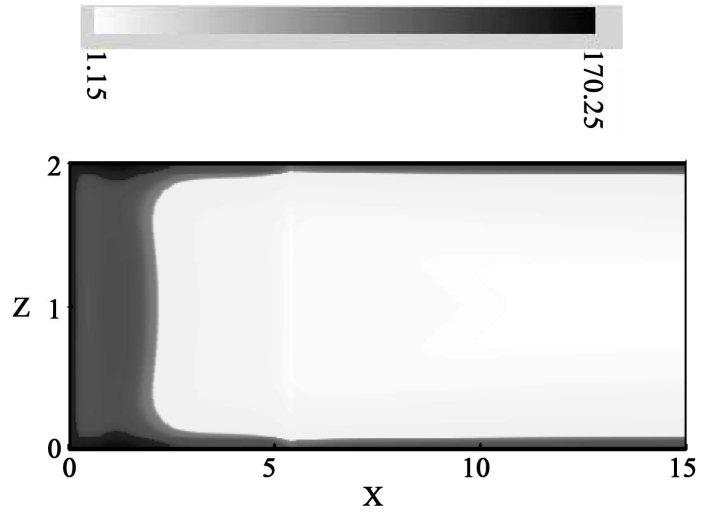

(a)

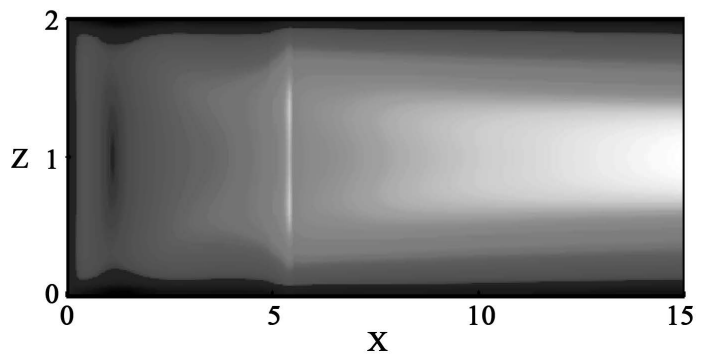

(b)

Figure 8. Distribution of the radiative Nusselt number contours on the bottom wall at different optical thickness, $R C=100, \omega=0.5$. (a) $\tau=0.01$; (b) $\tau=0.05$. 
Nusselt number. For more study about the effect of optical thickness on the thermal behavior of the convection-radiation system, the counters of total Nusselt number on the bottom wall for two different values of the optical thickness are plotted in Figure 9. It is seen that as the medium's ability to absorb and emit thermal radiation becomes greater at high values of the optical thickness, such systems have high values for the total Nusselt number.

In order to have another form of figures about the influence of optical thickness on the Nusselt number in convection-radiation system, the distributions of convective, radiative and total Nusselt numbers along the centerline on the bottom wall are plotted in Figures 10(a)-(c) for different value of the optical thickness. These figures show the same trend for variations of all types of Nusselt number as it was seen before in Figures 7-9.

\subsection{The Effect of Radiation-Conduction Parameter}

Radiation-conduction parameter (RC) is another one of the main parameters in the combined radiation-conduction systems, which show the relative importance of the radiation mechanism compared with its conduction counterpart. High value of RC parameter shows the radiation dominance in a thermal system. The effect of RC parameter on the contours of total Nusselt number is showed in Figure 11.

This figure illustrates that the total Nusselt number increases by increasing in RC parameter. This is due to this fact that under the effective presence of radiation mechanism at high values of $\mathrm{RC}$, the radiative Nusselt number gets increase that consequently leads to an increase in total Nusselt. Distribution of total Nusselt number along the bottom wall on the centerline is represented in Figure 12 for three different values of the RC parameter. This figure which is in consistence with the previous figure, clearly presents that how total Nusselt number affected by the variation of $\mathrm{RC}$ parameter.

To study the effect of radiation-conduction parameter $(\mathrm{RC})$ on the mean bulk temperature distribution along the duct Figure 13 is plotted. This figure shows that the mean bulk temperature increases along the duct because of both convection and radiation mechanisms. As it is seen from Figure 13, increase of RC parameter and consequently increase of radiation heat transfer mechanism causes an increase in the amount of the gas mean bulk temperature.

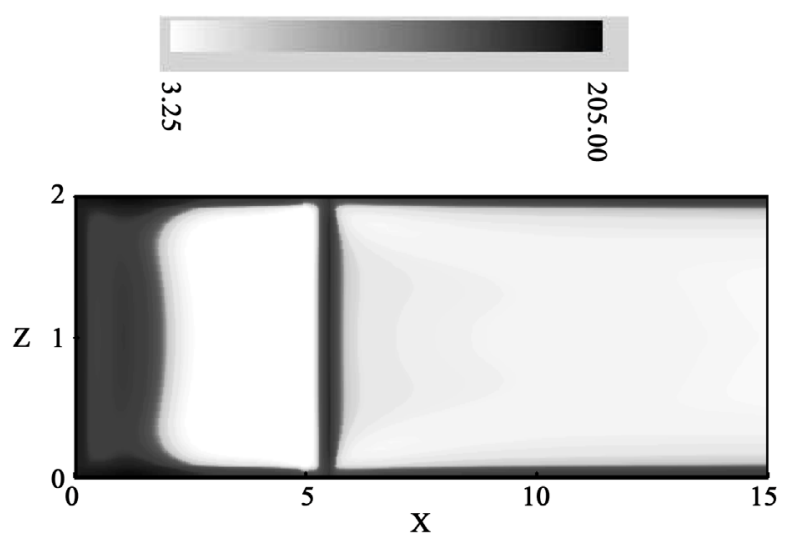

(a)

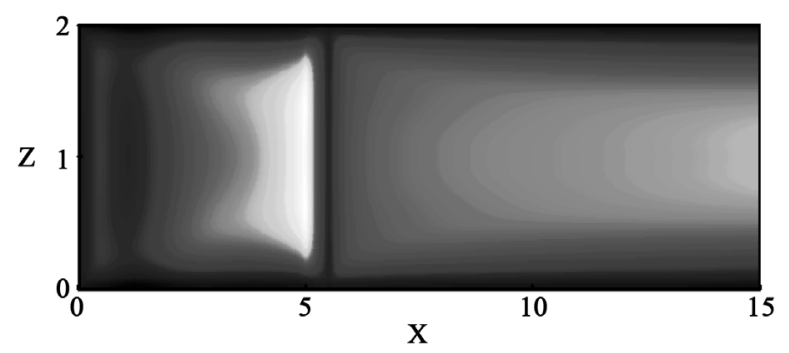

(b)

Figure 9. Distribution of the total Nusselt number contours on the bottom wall at different optical thickness, $R C=100, \omega=0.5$. (a) $\tau=0.01$; (b) $\tau=0.05$. 




(a)

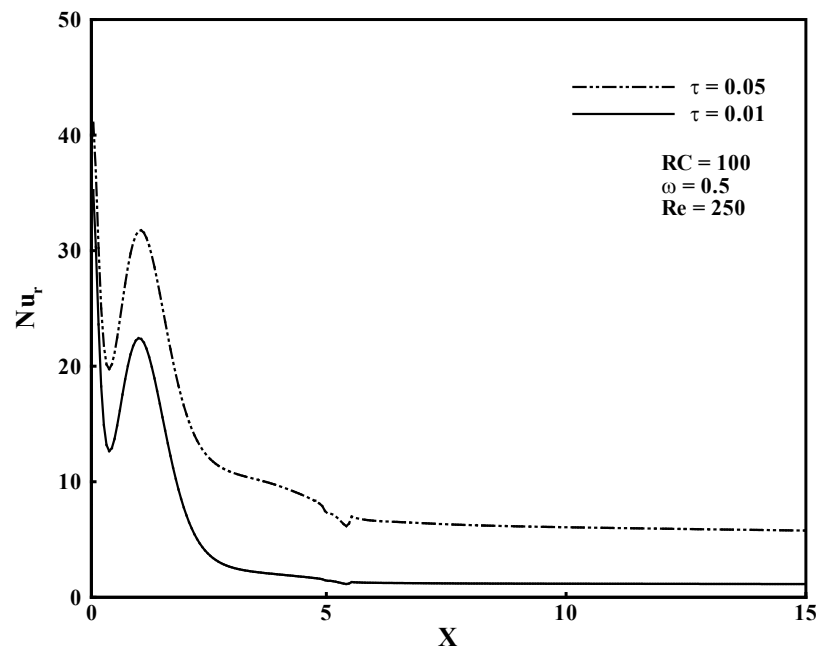

(b)

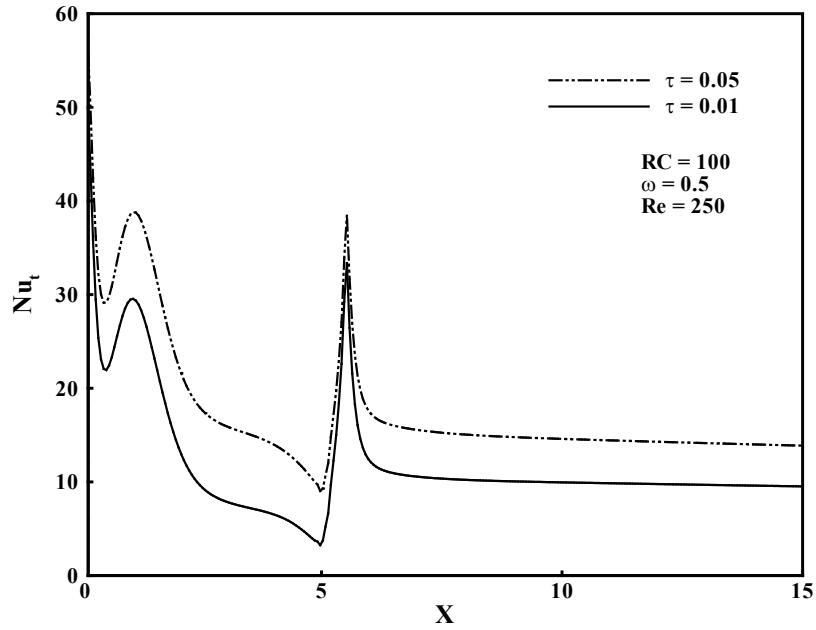

(c)

Figure 10. Effect of $\tau$ on the Nusselt number distribution along the centerline on the bottom wall. (a) Convective Nusselt number; (b) Radiative Nusselt number; (c) Total Nusselt number. 

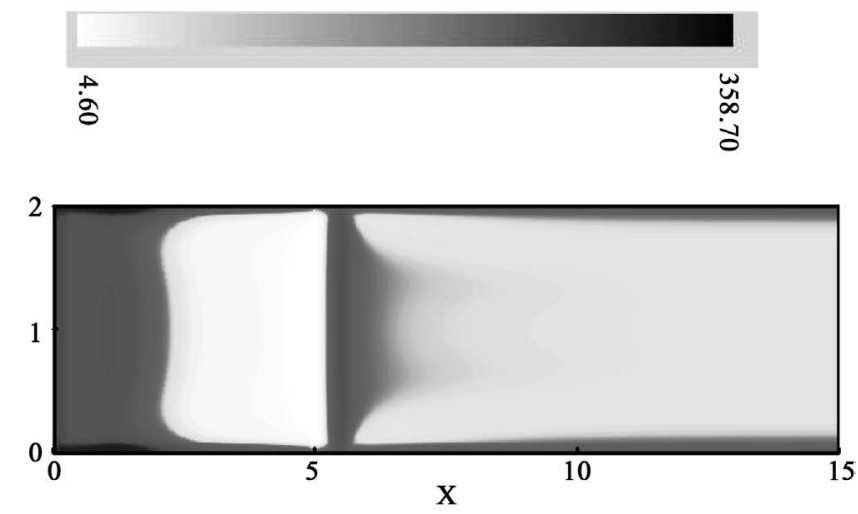

(a)

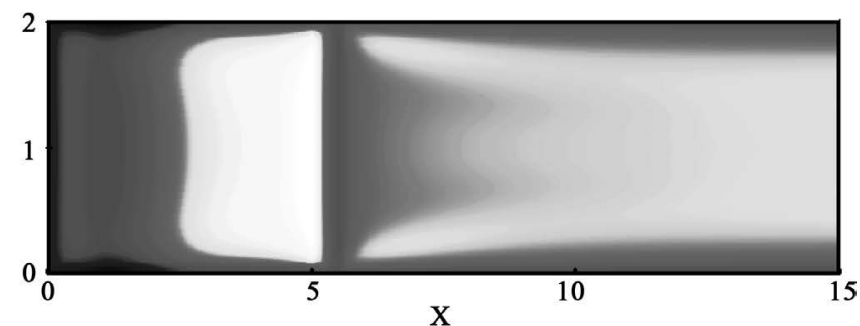

(b)

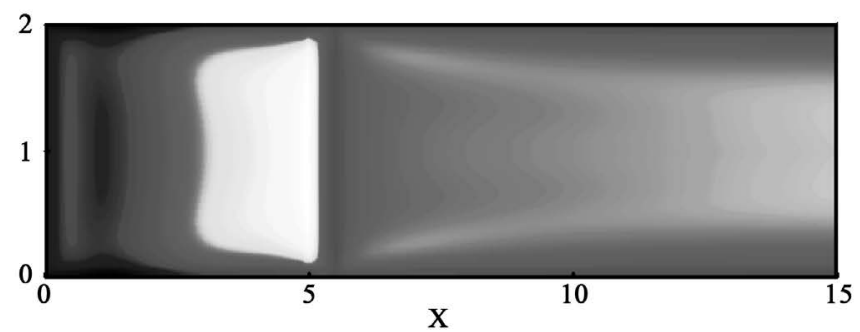

(c)

Figure 11. Effect of RC on the total Nusselt number contours along the bottom wall, $\omega=$ $0.5, \tau=0.005$. (a) $\mathrm{RC}=100$; (b) $\mathrm{RC}=200$; (c) $\mathrm{RC}=300$.

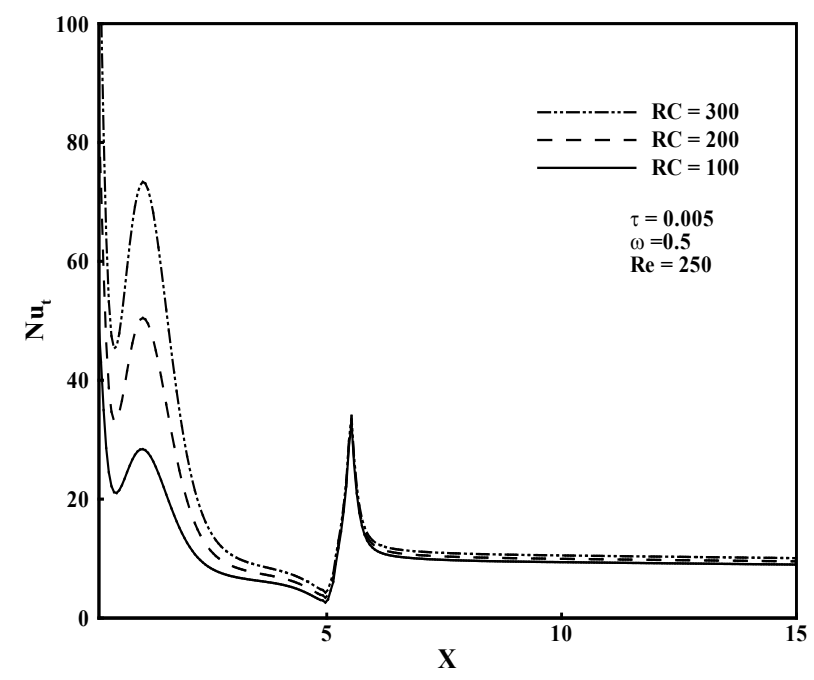

Figure 12. Effect of RC on the total Nusselt number distribution along the centerline on the bottom wall. 


\subsection{The Effect of Scattering Albedo}

The scattering albedo is an important parameter in radiating systems that can show the ability of participating medium to scatter thermal radiation. As it was mentioned before, scattering albedo, $\omega$, is defined as $\omega=\frac{\sigma_{s}}{\beta}$.

The extreme values of scattering albedo, i.e., $\omega=1.0$ and $\omega=0.0$ correspond to pure scattering and non-scattering cases, respectively. Therefore, the medium changes from pure absorption to pure scattering by increasing $\omega$ from 0 to 1 .

The effect of scattering albedo coefficient on the mean bulk temperature is presented in Figure 14. This figure show that the mean bulk temperature decreases by increasing in scattering albedo coefficient. Because, less radiative heat flux is converted to gas thermal energy in a pure scattering case compared to a pure absorption one. Besides, it can be found from Figure 14 that when the radiation term is omitted from the energy equation in

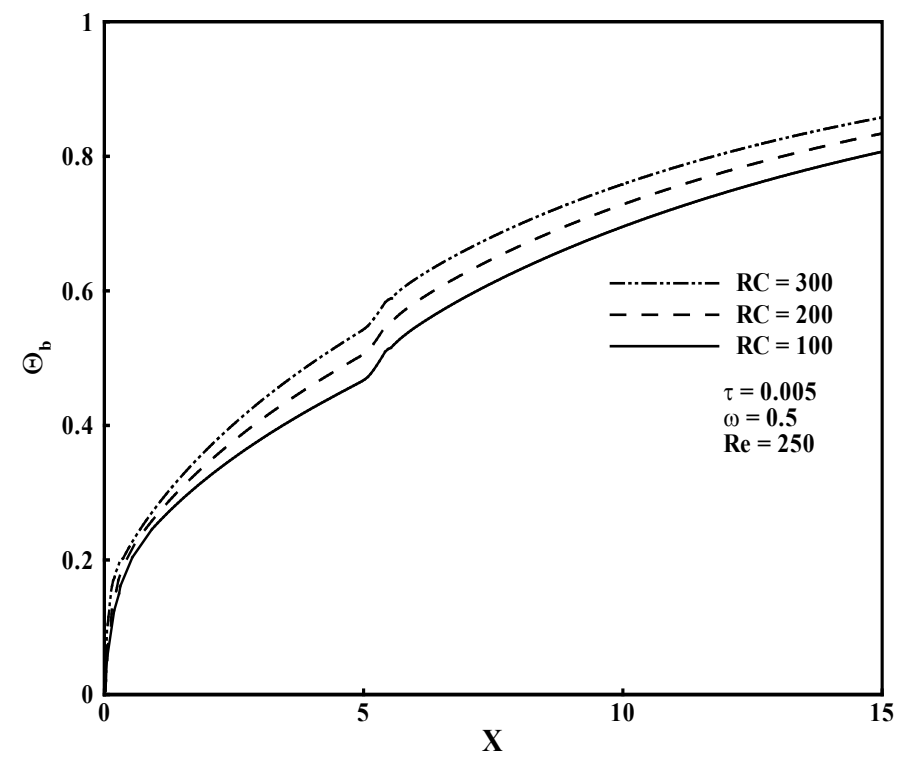

Figure 13. Effect of RC on the mean bulk temperature distribution along the duct.

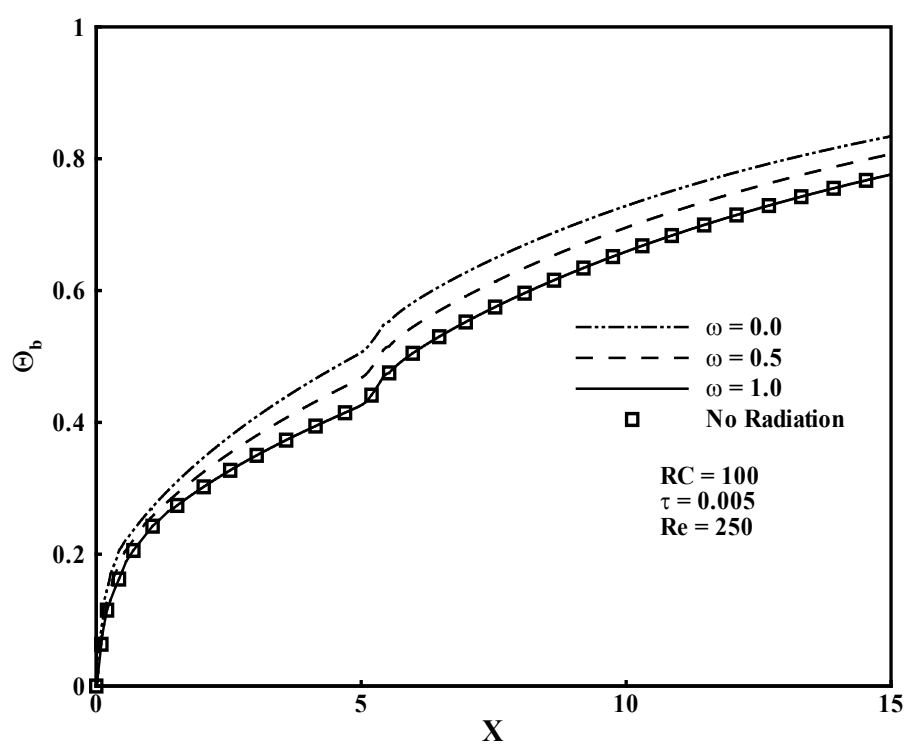

Figure 14. Effect of $\omega$ on the mean bulk temperature distribution along the duct. 
the case of no-radiation problems, the convective system has the same trend and behavior as pure scattering case with $\omega=1.0$.

\section{Conclusion}

Simulation of combined convection and radiation heat transfer over an inclined forward facing step (FFS) in a rectangular duct with constant step inclination angle in a 3-D horizontal duct is studied in this research work. The set of governing equations consisting of the continuity, momentum and energy is solved numerically by the CFD techniques in the Cartesian coordinate system. The blocked-off method is used in this study to simulate the incline surface of FFS. For calculating the radiative term in the energy equation, the RTE is solved by the DOM to obtain the distribution of radiant intensity inside the radiating medium. The effects of optical thickness, the radiation-conduction parameter and albedo coefficient on thermal behavior of the convective system are thoroughly explored by plotting the variations of Nusselt number (total, radiative and convective), and mean bulk temperature under different conditions.

\section{References}

[1] Vradis, G. and Nostrand, V.L. (1992) Laminar Coupled Flow Downstream an Asymmetric Sudden Expansion. Journal of Thermophysics Heat Transfer, 6, 288-295. http://dx.doi.org/10.2514/3.357

[2] Kondoh, T., Nagano, Y. and Tsuji, T. (1993) Computational Study of Laminar Heat Transfer Downstream of a Backward-Facing Step. International Journal of Heat and Mass Transfer, 36, 577-591. http://dx.doi.org/10.1016/0017-9310(93)80033-Q

[3] Erturk, E. (2008) Numerical Solutions of 2-D Steady Incompressible Flow over a Backward-Facing Step, Part I: High Reynolds Number Solutions. Computers \& Fluids, 37, 633-655. http://dx.doi.org/10.1016/j.compfluid.2007.09.003

[4] Atashafrooz, M., Gandjalikhan, S., Nassab, A. and Ansari, A.B. (2011) Numerical Study of Entropy Generation in Laminar Forced Convection Flow over Inclined Backward and Forward Facing Steps in a Duct. International Review of Mechanical Engineering, 5, 898-907.

[5] Armaly, B.F., Li, A. and Nie, J.H. (2003) Measurements in Three-Dimensional Laminar Separated Flow. International Journal of Heat and Mass Transfer, 46, 3573-3582. http://dx.doi.org/10.1016/S0017-9310(03)00153-4

[6] Beaudoin, J.F., Cadot, O., Aider, J.L. and Wesfreid, J.E. (2004) Three-Dimensional Stationary Flow over a Backward-Facing Step. European Journal of Mechanics B/Fluids, 23, 147-155. http://dx.doi.org/10.1016/j.euromechflu.2003.09.010

[7] Iwai, H., Nakabe, K. and Suzuki, K. (2000) Flow and Heat Transfer Characteristics of Backward-Facing Step Laminar Flow in a Rectangular Duct. International Journal of Heat and Mass Transfer, 43, 457-471. http://dx.doi.org/10.1016/S0017-9310(99)00140-4

[8] Nie, J.H. and Armaly, B.F. (2004) Convection in Laminar Three-Dimensional Separated Flow. International Journal of Heat and Mass Transfer, 47, 5407-5416. http://dx.doi.org/10.1016/j.ijheatmasstransfer.2004.07.030

[9] Iwai, H., Nakabe, K., Suzuki, K. and Matsubara, K. (2000) The Effects of Duct Inclination Angle on Laminar Mixed Convective Flows over a Backward-Facing Step. International Journal of Heat and Mass Transfer, 43, 473-485. http://dx.doi.org/10.1016/S0017-9310(99)00141-6

[10] Nie, J.H. and Armaly, B.F. (2002) Three-Dimensional Convective Flow Adjacent to Backward-Facing Step: Effects of Step Height. International Journal of Heat and Mass Transfer, 45, 2431-2438. http://dx.doi.org/10.1016/S0017-9310(01)00345-3

[11] Uruba, V., Jonas, P. and Mazur, O. (2007) Control of a Channel-Flow behind a Backward-Facing Step by Suction/Blowing. International Journal of Heat and Fluid Flow, 28, 665-672.

[12] Bahrami, A. and Nassab, S.A.G. (2010) Study of Entropy Generation in Laminar Forced Convection Flow over a Forward-Facing Step in a Duct. International Review of Mechanical Engineering, 4, 399-404.

[13] Abu-Mulaweh, H.I. (2003) A Review of Research on Laminar Mixed Convection Flow over Backward- and Forward-Facing Steps. International Journal of Thermal Sciences, 42, 897-909. http://dx.doi.org/10.1016/S1290-0729(03)00062-0

[14] Abu-Mulaweh, H.I. (2005) Turbulent Mixed Convection Flow over a Forward-Facing Step: The Effect of Step Heights. International Journal of Thermal Sciences, 44, 155-162. http://dx.doi.org/10.1016/j.ijthermalsci.2004.08.001

[15] Yilmaz, I. and Öztop, H.F. (2006) Turbulence Forced Convection Heat Transfer over Double Forward Facing Step Flow. International Communications in Heat and Mass Transfer, 33, 508-517. http://dx.doi.org/10.1016/j.icheatmasstransfer.2005.08.015 
[16] Chen, Y.T., Nie, J.H., Hsieh, H.T. and Sun, L.J. (2006) Three-Dimensional Convection Flow Adjacent to Inclined Backward-Facing Step. International Journal of Heat and Mass Transfer, 49, 4795-4803. http://dx.doi.org/10.1016/j.ijheatmasstransfer.2006.06.014

[17] Nassab, S.A.G., Moosavi, R. and Sarvari, S.M.H. (2009) Turbulent Forced Convection Flow Adjacent to Inclined forward Step in a Duct. International Journal of Thermal Sciences, 48, 1319-1326. http://dx.doi.org/10.1016/j.ijthermalsci.2008.10.003

[18] Bouali, H. and Mezrhab, A. (2006) Combined Radiative and Convective Heat Transfer in a Divided Channel. International Journal of Numerical Mathematics, 16, 84-106. http://dx.doi.org/10.1108/09615530610636973

[19] Azad, F.H. and Modest, M.F. (1981) Combined Radiation and Convection in Absorbing Emitting and Anisotropically Scattering Gas-Particulate Flow. International Journal of Heat Transfer, 24, 1681-1698. http://dx.doi.org/10.1016/0017-9310(81)90077-6

[20] Ansari, A.B. and Nassab, S.A.G. (2010) Thermal Characteristics of Convective Flows Encountered in a 2-D Backward Facing Step under the Effect of Radiative Heat Transfer. International Review of Mechanical Engineering, 4, 711-718.

[21] Atashafrooz, M. and Nassab, S.A.G. (2012) Numerical Analysis of Laminar Forced Convection Recess Flow with Two Inclined Steps Considering Gas Radiation Effect. Computers \& Fluids, 66, 167-176. http://dx.doi.org/10.1016/j.compfluid.2012.06.020

[22] Ansari, A.B. and Nassab, S.A.G. (2011) Numerical Analysis of Laminar Forced Convection Flow of a Radiating Gas over an Inclined Forward Facing Step. International Review of Mechanical Engineering, 5, 120-127.

[23] Atashafrooz, M. and Nassab, S.A.G. (2013) Simulation of Laminar Mixed Convection Recess Flow Combined with Radiation Heat Transfer. Iranian Journal of Science and Technology, 37, 71-75.

[24] Ko, M. and Anand, N.K. (2008) Three-Dimensional Combined Convective-Radiative Heat Transfer over a Horizontal Backward-Facing Step-A Finite Volume Method. Numerical Heat Transfer, 54, 109-129. http://dx.doi.org/10.1080/10407780802025721

[25] Atashafrooz, M. and Nassab, S.A.G. (2012) Simulation of Three-Dimensional Laminar Forced Convection Flow of a Radiating Gas over an Inclined Backward-Facing Step in a Duct under Bleeding Condition, Institution of Mechanical Engineers, Part C. Journal of Mechanical Engineering Science, 227, 332-345. http://dx.doi.org/10.1177/0954406212447657

[26] Atashafroozand, M. and Nassab, S.A.G. (2012) Combined Heat Transfer of Radiation and Forced Convection Flow of Participating Gases in a Three-Dimensional Recess. Journal of Mechanical Science and Technology, 26, 3357-3368. http://dx.doi.org/10.1007/s12206-012-0804-x

[27] Chiu, H.C., Jang, J.H. and Yan, W.M. (2007) Mixed Convection Heat Transfer in Horizontal Rectangular Ducts with Radiation Effects. International Journal of Heat and Mass Transfer, 50, 2874-2882. http://dx.doi.org/10.1016/j.ijheatmasstransfer.2007.01.010

[28] Chiu, H.C. and Yan, W.M. (2008) Mixed Convection Heat Transfer in Inclined Rectangular Ducts with Radiation Effects. International Journal of Heat and Mass Transfer, 51, 1085-1094. http://dx.doi.org/10.1016/j.ijheatmasstransfer.2007.05.009

[29] Modest, M.F. (2003) Radiative Heat Transfer. 2nd Edition, McGraw-Hill, New York.

[30] Keshtkar, M.M. and Nassab, S.A.G. (2009) Theoretical Analysis of Porous Radiant Burners under 2-D Radiation Field Using Discrete Ordinates Meth. Radiative Transfer, 110, 1894-1907.

[31] Patankar, S.V. and Spalding, D.B. (1972) A Calculation Procedure for Heat, Mass and Momentum Transfer in Three-Dimensional Parabolic Flows. International Journal of Heat and Mass Transfer, 15, 1787-1806. http://dx.doi.org/10.1016/0017-9310(72)90054-3

[32] Patankar, S.V. (1981) Numerical Heat Transfer and Fluid Flow. Taylor \& Francis, Philadelphia. 